\title{
Interferometric imaging of multiples in an RTM approach
}

Alison Malcolm*, Earth Resources Laboratory and Department of Earth, Atmospheric and Planetary Sciences, MIT and Maarten de Hoop, Department of Mathematics and Department of Earth Sciences, Purdue University

\section{SUMMARY}

It is well known that reverse-time migration is capable of correctly imaging multiply scattered energy. To do this, one of the interfaces from which the waves scatter must be included in the background velocity model. In a one-way framework this requirement is avoided by iteratively forming images of higher-order scattered waves. These techniques use an image made with singly scattered waves to estimate the locations of some of the reflection points in a multiply-scattered wave, from which the location of an additional scattering point is determined through standard imaging techniques. This removes the requirement that a single multiple-generating interface be identified Here we extend this technique to reverse-time migration using results from several recent studies linking standard and extended imaging conditions to interferometry. This results in a method to generate images with multiply-scattered waves using the full-waveform imaging techniques of reverse-time migration and the iterative imaging formulations of scattering series in a one-way framework.

\section{INTRODUCTION}

When imaging complicated geologic structures, the single scattering assumption may restrict the ability of standard imaging algorithms to form an image throughout the domain. Several authors have suggested methodologies for including multiply scattered waves in the imaging procedure to mitigate this problem, for example Jin et al. (2006); Malcolm et al. (2009) in a one-way framework, and Farmer et al. (2006); Jones et al. (2007) in reverse-time migration (RTM). In the RTM case, including multiply-scattered waves in the imaging procedure requires the specific inclusion of a reflector in the velocity model; data requirements for this approach to avoid artifacts is discussed specifically in Mittet $(2002,2006)$. By contrast, in the one-way framework proposed in Malcolm et al. (2009), an estimate of the image of the subsurface is used to include a single reflection in the back-propagation, when the imaging is done within the context of a scattering series such as those discussed in de Hoop (1996); Malcolm et al. (2005) or Weglein et al. (2003). This latter formulation has the advantage of being able to image with multiply scattered waves without explicitly defining a single reflector, but the formulation has so far been restricted to one-way imaging.

To extend these image-driven nonlinear imaging techniques to RTMtype imaging algorithms, we rely on relationships between seismic interferometry (see e.g. de Hoop and de Hoop (2000); Wapenaar and Fokkema (2006); Snieder et al. (2007) and references therein) and migration. That there is a relationship between interferometry and migration has been discussed by several authors. One could make the argument that Esmersoy and Oristaglio (1988) was the first to discuss this relationship, though his work preceeded the current interest in interferometry. More recently, van Manen et al. (2006); Thorbecke and Wapenaar (2007) discuss the relationship between migration resolution and interferometry. In the context developed here, the most closely related discussions are those of extended images in Vasconcelos et al. (2009) and the discussion of migration in Stolk et al. (2009b,a). In the following sections, we will develop and expand on the relationship between interferometry and imaging, as discussed in Vasconcelos et al. (2009) and show how this relationship can be exploited to do image-driven migration of multiply-scattered waves.

\section{BORN APPROXIMATION AND INTERFEROMETRY}

We follow closely Stolk et al. (2009a), in which the relationship between interferometry and reverse-time migration is made explicit in their equation 6.5. Our goal is different, however, since we seek to extract the Green's function between two points in the subsurface from the data recorded at the surface, rather than forming an image per se. The resulting data are suitable for imaging via an RTM from below. To introduce notation and methods, we first discuss extracting the Green's function between two points in the subsurface using the Born approximation.

We begin from the standard first-order system of equations governing acoustic wave propagation

$$
\begin{aligned}
\kappa \partial_{t} p-\nabla \cdot \mathbf{v} & =q, \\
\rho \partial_{t} \mathbf{v}-\nabla p & =\mathbf{f}
\end{aligned}
$$

where $p$ is the pressure, $\mathbf{v}$ is the velocity, $\rho$ is the density and $\kappa$ is the compressibility. The two sources, $q$ and $\mathbf{f}$ are the volume source density of injection rate and the volume source density of force, respectively. Here, we use bold type to indicate a vector quantity and bold capital letters to indicate a tensor quantity. In general, the solution to this system is characterized by the four Green's functions $g^{p q}$ and $\mathbf{g}^{v q}$ for a point source in $q$ and $\mathbf{g}^{p f}, \mathbf{G}^{v f}$ for a point source in $\mathbf{f}$. Here, to mimic the pressure recordings in typically made in marine data acquisitions, we instead introduce the scalar-wave green's function $g$, which is the pressure field, $p$, subject to the conditions

$$
\begin{aligned}
\mathbf{f} & =0 \\
\rho(x) \partial_{t} q(x, t) & =\delta\left(x-x^{\prime}\right) \delta(t) .
\end{aligned}
$$

Under the Born Approximation, assuming constant density, we define the contrast sources

$$
\begin{aligned}
q^{s c}(x, t, s) & =-\delta \kappa(x) \partial_{t} p^{0}(x, t, s) \\
\mathbf{f}^{s c}(x, t, s) & =0
\end{aligned}
$$

which generate the singly scattered field, from the incident field $p^{0}$ at any point in the subsurface at which there is a jump in compressibility. If we assume, in addition, that there are no sources on the boundary (at the surface in this case), then we have in addition that

$$
\mathbf{v}^{s c}(x, t, s)=\rho^{-1} \partial_{t}^{-1} \nabla p^{s c}(x, t, s)
$$

for points $x$ on the surface, $\partial \Omega$ bounding the volume of interest, $\Omega$. With these simplifications, we obtain the following expression for the scattered pressure field under the Born approximation,

$$
\hat{p}^{s c}(x, \omega, s)=\omega^{2} \int_{\Omega} \hat{g}_{0}\left(x, \omega, x^{\prime}\right) \hat{p}^{0}(x, \omega, s) \delta c^{-2}\left(x^{\prime}\right) d V\left(x^{\prime}\right),
$$

where the ${ }^{\wedge}$ indicates Fourier transformation with respect to time and the subscript 0 on $\hat{g}_{0}$ indicates that this Green's function is assumed to be in a smooth background devoid of scatterers, and $\delta c(x)^{-2}=\rho \delta \kappa$. We now rewrite this expression in a form that makes clear the connection with the interferometric Green's function between two points along the wavepath. 


\section{Interferometric Imaging of Multiples}

As in many of the derivations of interferometry (e.g. Wapenaar and Fokkema (2006)), our derivation also relies on the representation theorem and the reciprocity relations that follow from it. Specifically, we use the following relation for the scattered field

$$
\begin{aligned}
\hat{p}^{s c}\left(x^{\prime}, \omega, s\right)=\int_{\partial \Omega}[ & \mathbf{n}(x) \cdot \hat{\mathbf{v}}^{s c}(x, \omega, s) \overline{\hat{g}^{p q}\left(x, \omega, x^{\prime}\right)} \\
& \left.+\hat{p}^{s c}(x, \omega, s) \mathbf{n}(x) \cdot \overline{\hat{\mathbf{g}}^{v q}\left(x, \omega, x^{\prime}\right)}\right] d A(x)
\end{aligned}
$$

given in e.g. de Hoop and de Hoop (2000), where $\mathbf{n}$ is the outward pointing normal and the - denotes the adjoint. Substituting equation 8 for the scattered field on the right-hand side as well as the Born approximation via 6 into this expression gives

$$
\begin{aligned}
\hat{p}^{s c}\left(x^{\prime}, \omega, s\right)= & \omega^{2} \int_{\Omega} \hat{p}_{0}\left(x^{\prime \prime}, \omega, s\right) \delta c^{-2}\left(x^{\prime \prime}\right) \\
& \int_{\partial \Omega}\left[\mathbf{n}(x) \nabla_{x} \hat{g}_{0}\left(x, \omega, x^{\prime \prime}\right) \overline{\hat{g}_{0}\left(x, \omega, x^{\prime}\right)}\right. \\
+ & \left.\hat{g}_{0}\left(x, \omega, x^{\prime \prime}\right) \mathbf{n}(x) \overline{\nabla_{x} \hat{g}_{0}\left(x, \omega, x^{\prime}\right)}\right] d A(x) d V\left(x^{\prime \prime}\right),
\end{aligned}
$$

where we have used the smooth-background Green's function $g_{0}$ because we assume that all of the scattering is accounted for explicitly via the Born approximation; we will relax this requirement in the next section. In equation 10 , we recognize the inner surface integral as the usual intereferometric Green's function, $\hat{h}\left(x^{\prime}, \omega, x^{\prime \prime}\right)=$ $2 \operatorname{IIm}\left[\hat{g}\left(x^{\prime}, \omega, x^{\prime \prime}\right)\right]$. As is well known, the interferometric recovery is perfect only if there are sources completely surrounding the two receivers. In the notation above, this means that the integration over $x$ must be over a surface surrounding $x^{\prime \prime}$ and $x^{\prime}$; this is not in general the case and so we will use $\hat{h}^{\Sigma_{s}}$ to indicate that this Green's function is constructed by integrating only over the receiver array for the source $s$. This then simplifies the expression for the scattered field to

$$
\hat{p}^{s c}\left(x^{\prime}, \omega, s\right)=\omega^{2} \int_{\Omega} \hat{p}_{0}\left(x^{\prime \prime}, \omega, s\right) \delta c^{-2}\left(x^{\prime \prime}\right) \hat{h}_{0}^{\Sigma_{s}}\left(x^{\prime}, \omega, x^{\prime \prime}\right) d V\left(x^{\prime \prime}\right) .
$$

Having derived expression 11 for the scattered field in terms of the interferometric Green's function, we now show how this Green's function could be extracted in an RTM framework. We begin by applying a nearly-standard imaging principal to this expression for the scattered field, that is the correlation of the back-propagated data (here represented by the scattered field $\left.\hat{p}^{s c}\left(x^{\prime}, \omega, s\right)\right)$, with the source-side incident wavefield $\hat{p}^{0}(s, \omega, x)$ and integration over sources. Applying these steps to 11, without enforcing that the fields be evaluated at the same subsurface points, as in the extended imaging conditions of e.g. Sava and Vasconcelos (2009); Vasconcelos et al. (2009); Esmersoy and Oristaglio (1988) and references therein, gives

$$
\begin{aligned}
& \int_{\partial \Omega} \hat{p}^{0}(s, \omega, y) \overline{\hat{p}^{s c}\left(x^{\prime}, \omega, s\right)} d A(s) \\
&=\omega^{2} \int_{\partial \Omega} \hat{p}^{0}(s, \omega, y) \int_{\Omega}\left\{\overline{\hat{h}_{0}^{\Sigma_{s}}\left(x^{\prime}, \omega, x^{\prime \prime}\right)}\right. \\
&\left.\overline{\hat{p}^{0}\left(x^{\prime \prime}, \omega, s\right)} \delta c^{-2}\left(x^{\prime \prime}\right)\right\} d V\left(x^{\prime \prime}\right) d A(s) .
\end{aligned}
$$

If we assume complete source coverage, then the source integration results in $\delta\left(x-x^{\prime \prime}\right)$ leading to the result

$$
\int_{\partial \Omega} \hat{p}^{0}(s, \omega, y) \hat{p}^{s c}\left(x^{\prime}, \omega, s\right) d A(s)=-\omega^{2} \hat{h}^{\Sigma_{s}}\left(x^{\prime}, \omega, y\right) \delta c^{-2}(y) .
$$

From this expression we see that we can extract the Green's function with the following steps,

- forward propagate the source wavefield, $p^{0}$ for source position $s$, to the point $y$

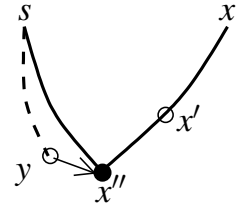

Figure 1: We first extract the Green's function between $x^{\prime}$ and $y$; for this to be nonzero in the Born approximation, $y=x^{\prime \prime}$.

- backward propagate the data, $p^{s c}$, also for the source $s$ to $x^{\prime}$

- cross-correlate the resulting fields

- multiply the result by $\omega^{-2}$ and the reflectivity, $\delta c^{2}$, at the point $y$ to which the source-side wavefield has been propagated

- sum the result over all sources.

It is important to note that this construction is only possible when $y$ corresponds to an actual scatterer in the subsurface. Extracting the Green's function between two arbitrary points is discussed in the next section. From this result, however, we can already anticipate that the Green's function could be extracted between two scattering points, allowing imaging with internal multiples. In addition, we emphasize that this recovery will not be exact because of the limited coverage in both source and receiver.

\section{RECOVERING THE GREEN'S FUNCTION BETWEEN ARBI- TRARY POINTS}

In the previous section, we derived that an interferometric Green's function can be extracted between an arbitrary point and the scattering point in the Born approximation, as illustrated in Figure 1. As noted by Wapenaar (2009), even for a single point diffractor the Born approximation is not adequate for extracting the scattered field Green's function. To begin to go beyond this, as well as to set the stage for the multiply-scattered case, we now modify the result of the previous section, showing how the Green's function can be extracted between two arbitrary points, as is expected from classic interferometry.

We first generalize the Born scattered field, 8 to

$$
\hat{p}^{s c}(x, \omega, s)=\int_{\Omega} \hat{g}\left(x, \omega, x^{\prime}\right) \hat{p}^{0}(x, \omega, s) d V\left(x^{\prime}\right)
$$

where we now allow for scattering contained within the Green's function $\hat{g}$; if we use the Born approximation for this Green's function, we would recover exactly 8 .

As in the previous section, we again introduce the representation theorem to rewrite 14 as

$$
\begin{gathered}
\hat{p}^{s c}\left(x^{\prime \prime}, \omega, s\right)=\int_{\Omega}\left\{\hat{p}^{0}\left(x^{\prime}, \omega, s\right) d V\left(x^{\prime}\right)\right. \\
\left.\int_{\partial \Omega} \mathbf{n}(x) \cdot\left[\nabla_{x} \hat{g}\left(x, \omega, x^{\prime}\right) \overline{\hat{g}\left(x^{\prime \prime}, \omega, x\right)}+\hat{g}\left(x, \omega, x^{\prime}\right) \overline{\nabla_{x} \hat{g}\left(x^{\prime \prime}, \omega, x\right)}\right]\right\} \\
\quad=\int_{\Omega} d V\left(x^{\prime}\right) \hat{p}^{0}\left(x^{\prime}, \omega, s\right) \hat{h}\left(x^{\prime \prime}, \omega, x^{\prime}\right)
\end{gathered}
$$

from which we see that we can extract the interferometric Green's function $h$ between two arbitrary points, $x^{\prime}$ and $x^{\prime \prime}$ by cross-correlating the source wavefield, $p^{0}$ evaluated at $x^{\prime}$ and the data, back propagated to $x^{\prime \prime}$. In this formulation, we would now expect to extract the Green's function containing both singly and multiply-scattered arrivals as illustrated in Figure 2. 


\section{Interferometric Imaging of Multiples}

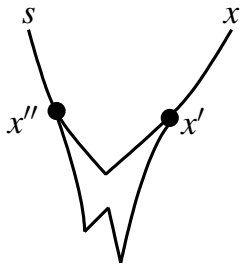

Figure 2: In recovering the Green's function between $x^{\prime}$ and $x^{\prime \prime}$ in the formulation given in equation 15 , both arrivals pictured would be expected to be recovered.

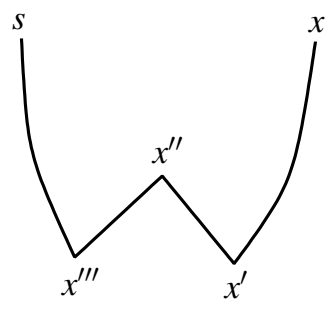

Figure 3: Notation for multiple scattering.

\section{THE GREEN'S FUNCTION BETWEEN TWO SCATTERING POINTS}

Now that we have established that the Green's function can be extracted between arbitrary points, we will now setup a framework in which the Green's function is estimated between two scattering points, thus turning an internal multiple into a primary. This is, of course, what has been used to image subsalt using surface sources in a horizontal well in e.g. Vasconcelos et al. (2008). To begin, we model an internal multiple as the third term of the Lippmann-Schwinger scattering series, using the notation illustrated in Figure 3

$$
\begin{gathered}
\hat{p}_{3}^{s c}(x, \omega, s)=\omega^{6} \int_{\Omega} \int_{\Omega} \int_{\Omega}\left\{\hat{g}\left(x, \omega, x^{\prime}\right) \delta c^{-2}\left(x^{\prime}\right)\right. \\
\hat{g}\left(x^{\prime}, \omega, x^{\prime \prime}\right) \delta c^{-2}\left(x^{\prime \prime}\right) \hat{g}\left(x^{\prime \prime}, \omega, x^{\prime \prime \prime}\right) \delta c^{-2}\left(x^{\prime \prime \prime}\right) \\
\left.\hat{p}^{0}\left(x^{\prime \prime \prime}, \omega, s\right)\right\} d V\left(x^{\prime \prime \prime}\right) d V\left(x^{\prime \prime}\right) d V\left(x^{\prime}\right),
\end{gathered}
$$

this equation is derived in a one-way framework in Malcolm et al. (2005); another discussion of a related scattering series can be found in Weglein et al. (2003). We have written an internal multiple in this form to demonstrate the ability of interferometry to simplify the wavefield and thus allow for the application of a multiple-targetted imaging procedure. To this end, we now consider evaluating the field in 16 at the first scattering point, $x^{\prime}$,

$$
\begin{gathered}
\hat{p}_{3}^{s c}\left(x^{\prime}, \omega, s\right)=\omega^{6} \delta c^{-2}\left(x^{\prime}\right) \int_{\Omega} d V\left(x^{\prime \prime}\right) \int_{\Omega}\left\{\hat{g}\left(x^{\prime}, \omega, x^{\prime \prime}\right)\right. \\
\left.\delta c^{-2}\left(x^{\prime \prime}\right) \hat{g}\left(x^{\prime \prime}, \omega, x^{\prime \prime \prime}\right) \delta c^{-2}\left(x^{\prime \prime \prime}\right) \hat{p}^{0}\left(x^{\prime \prime \prime}, \omega, s\right)\right\} d V\left(x^{\prime \prime \prime}\right) .
\end{gathered}
$$

This field would, in general, be estimated by time reversing and back propagating the data into the subsurface; in order for this to result in a perfect estimate of the field at $x^{\prime}$, full receiver coverage must be assumed, we suppress this in the notation, but note that the failure of this assumption will introduce some error.

We now form a new scattered field, $\mathscr{P}$, by multiplying 17 by the inverse of the scattering operator $\left(\omega^{-2} \delta c^{2}\right)$ at $x^{\prime}$

$$
\hat{\mathscr{P}}_{3}^{s c}\left(x^{\prime}, \omega, s\right)=\omega^{-2} \delta c^{2}\left(x^{\prime}\right) \hat{p}_{3}^{s c}\left(x^{\prime}, \omega, s\right) .
$$

With this definition, equation 17 gives

$$
\begin{aligned}
\hat{\mathscr{P}}_{3}^{s c}\left(x^{\prime}, \omega, s\right) & \\
= & \omega^{2} \int_{\Omega} \hat{g}\left(x^{\prime}, \omega, x^{\prime \prime \prime}\right) \delta c^{-2}\left(x^{\prime \prime \prime}\right) \hat{p}^{0}\left(x^{\prime \prime \prime}, \omega, s\right) d V\left(x^{\prime \prime \prime}\right) \\
= & \omega^{2} \int_{\Omega} \delta c^{-2}\left(x^{\prime \prime \prime}\right) \hat{p}^{0}\left(x^{\prime \prime \prime}, \omega, s\right)\{ \\
& \int_{\partial \Omega} \mathbf{n}(x) \cdot\left[\nabla_{x} \hat{g}\left(x, \omega, x^{\prime \prime \prime}\right) \hat{g}\left(x, \omega, x^{\prime}\right)\right. \\
& \left.\left.+\hat{g}\left(x, \omega, x^{\prime \prime \prime}\right) \overline{\nabla_{x} \hat{g}\left(x, \omega, x^{\prime}\right)}\right] d A(x)\right\} d V\left(x^{\prime \prime \prime}\right) \\
= & \omega^{2} \int_{\Omega} \delta c^{-2}\left(x^{\prime \prime \prime}\right) \hat{p}^{0}\left(x^{\prime \prime \prime}, \omega, s\right) \hat{h}\left(x^{\prime}, \omega, x^{\prime \prime \prime}\right) d V\left(x^{\prime \prime \prime}\right),
\end{aligned}
$$

where we have made the same assumption as in the previous section, that the Green's function does include the scattering.

Comparing with equation 15 , we notice that this field can then be written in the form

$$
\hat{\mathscr{P}}_{3}^{s c}\left(x^{\prime}, \omega, s\right)=\omega^{2} \int_{\Omega} \hat{p}_{1}^{s c}\left(x^{\prime}, \omega, x^{\prime \prime \prime}\right) \delta c^{-2}\left(x^{\prime \prime \prime}\right) \hat{p}^{0}\left(x^{\prime \prime \prime}, \omega, s\right) d V\left(x^{\prime \prime \prime}\right)
$$

where we have assumed a point source in the substitution of $\hat{p}_{1}^{s c}$.

From 19 we now proceed, along the same lines as in 13 to extract the interferometric Green's function for the triple scattering case resulting in

$$
\hat{h}\left(x^{\prime}, \omega, x^{\prime \prime \prime}\right)=\int_{\partial \Omega} \overline{\hat{\mathscr{P}}_{3}^{s c}\left(x^{\prime}, \omega, s\right)} \delta c^{2}\left(x^{\prime \prime \prime}\right) \omega^{-2} \hat{p}^{0}\left(x^{\prime \prime \prime}, \omega, s\right) d A(s),
$$

which is just the extended image as in e.g. Vasconcelos et al. (2009). To make this relation symmetric in source and receiver, we define $\hat{\mathscr{P}}^{0}$ as

$$
\hat{\mathscr{P}}^{0}\left(x^{\prime \prime \prime}, \omega, s\right)=\omega^{-2} \delta c^{2}\left(x^{\prime \prime \prime}\right) \hat{p}^{0}\left(x^{\prime \prime \prime}, \omega, s\right)
$$

reducing equation 21 to

$$
\hat{h}\left(x^{\prime}, \omega, x^{\prime \prime \prime}\right)=\int_{\partial \Omega} \overline{\hat{\mathscr{P}}_{3}^{s c}\left(x^{\prime}, \omega, s\right)} \hat{\mathscr{P}}^{0}\left(x^{\prime \prime \prime}, \omega, s\right) d s,
$$

From this form, we see that the interferometric Green's function is directly related to the cross-correlation of the forward propagated source field and back propagated data field, each scaled by the reflectivity at the virtual source/receiver location. This opens up the possibility of imaging with multiply-scattered waves in an iterative manner via the following steps

- perform a standard RTM

- define a multiple-generating zone; this could be the entire region or simply surrounding a single reflector

- for strong reflectivities in this zone, compute the interferometric Green's function $h$ between appropriate virtual source and receiver positions; choose points where the reconstructed primaries would be expected to illuminate the structures of interest from below and there is strong reflectivity

- use a standard RTM 'upside-down' to image with the reconstructed primaries.

This procedure is illustrated in Figure 4. 
Interferometric Imaging of Multiples

(a)

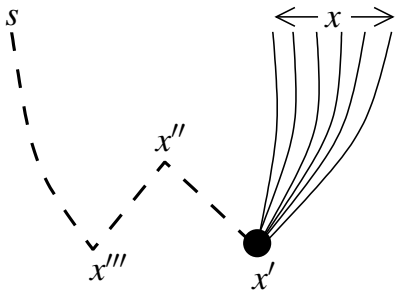

(b)
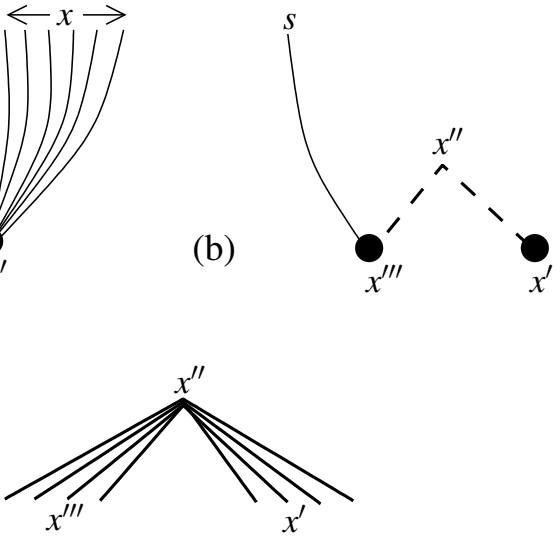

(d) (c)

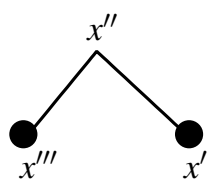

(e)

Figure 4: An illustration of the proposed imaging algorithm. (a) For a single shot, the data are time-reversed and back-propagated to the point $x^{\prime}$. (b) The same shot, $s$, is then forward propagated to $x^{\prime \prime \prime}$. (c) The resulting fields from (a) and (b) are cross-correlated and summed over all sources, resulting in a pseudo-data point in an upside-down geometry. (d) This is repeated for several virtual source/receiver locations. (e) The resulting data are used to image at a range of $x^{\prime \prime}$.

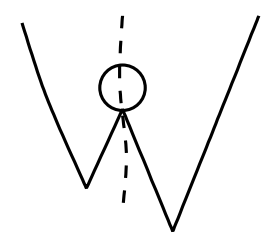

Figure 5: When imaging the circular scatterer, multiples image the structure without passing through it whereas zero-offset waves must pass through the object in order to image it.

\section{DISCUSSION}

In Vasconcelos et al. (2009) it had already been noted that the Green's function between two subsurface points could be estimated with an interferometry-like calculation, similar to that shown in the second section here. In subsequent sections, we have gone beyond this to show explicitly how these ideas can be exploited to develop imaging algorithms for first-order internal multiples. Vasconcelos (2008) discussed the relationship between Claerbout's classic imaging condition (Claerbout, 1985) and the zero-offset scattered Green's function recovered through interferometry. This work can also be understood in that context, in that it would be possible to recover directly the zerooffset Green's function, as a function of time, and thus to estimate, for example, the distance from the virtual source/receiver point to the reflector of interest. This would preclude, however, the use of multiply scattered waves to image structures without passing through them, as illustrated in Figure 5.

\section{CONCLUSIONS}

We have derived an equivalence between interferometry and reversetime migration in the context of imaging with multiply scattered waves. This establishes two key points: (i) similar results should be expected when imaging with multiples whether those multiples are used to reconstruct primaries as in the virtual source method or included directly in a reverse time migration and (ii) iterative imaging, in which an image, rather than a picked reflector, is used to generate multiple scattering is possible in an RTM framework. The first point is contingent on having a good model of the overburden, which is the main advantage of using receivers in a horizontal well, for example, along with the virtual source method to image beneath complicated overburden structures. The combination of numerical back-propagation and the replacement of the horizontal well with a reflector makes the method presented here cost effective when the overburden is less difficult to determine. The ability to image without including hard reflectors in the background model has several advantages including ease of computation and the ability to separate images made with different order scattering, allowing for more detailed interpretations of the different images.

\section{ACKNOWLEDGMENTS}

This work has benifitted greatly from discussions with Ivan Vasconcelos and Bjørn Ursin. AM is grateful for funding this project has received from Total and from the sponsors of the Earth Resources Laboratory. MVdH was supported in part by the members of the GeoMathematical Imaging Group. 


\section{EDITED REFERENCES}

Note: This reference list is a copy-edited version of the reference list submitted by the author. Reference lists for the 2010 SEG Technical Program Expanded Abstracts have been copy edited so that references provided with the online metadata for each paper will achieve a high degree of linking to cited sources that appear on the Web.

\section{REFERENCES}

Claerbout, J. F., 1985, Imaging the earth's interior: Blackwell Scientific Publications, Inc.

de Hoop, M. V., 1996, Generalization of the Bremmer coupling series: Journal of Mathematical Physics, 37, no. 7, 3246-3282, doi:10.1063/1.531566.

de Hoop, M. V. and A. T. de Hoop, 2000, Wavefield reciprocity and optimization in remote sensing: Proc. R. Soc. Lond. A (Mathematical, Physical and Engineering Sciences), 456, 641-682.

Esmersoy, C., and M. Oristaglio, 1988, Reverse-time wave-field extrapolation, imaging, and inversion: Geophysics, 53, 920-931, doi:10.1190/1.1442529.

Farmer, P. A., I. F. Jones, H. Zhou, R. I. Bloor, and M. C. Goodwin, 2006, Application of reverse time migration to complex imaging problems: First Break, 24, 65-73.

Jin, S., S. Xu, and D. Walraven, 2006, One-return wave equation migration: Imaging of duplex waves: SEG Technical Program Expanded Abstracts, 25, no. 1, 2338-2342, doi:10.1190/1.2370003.

Jones, I. F., M. C. Goodwin, I. D. Berranger, H. Zhou, and P. A. Farmer, 2007, Application of anisotropic 3D reverse time migration to complex North Sea imaging: SEG Technical Program Expanded Abstracts, 26, no. 1, 2140-2144, doi:10.1190/1.2792911.

Malcolm, A., B. Ursin, and M. de Hoop, 2009, Seismic imaging and illumination with internal multiples: Geophysical Journal International, 176, no. 3, 847-864, doi:10.1111/j.1365-246X.2008.03992.x.

Malcolm, A. E., M. V. de Hoop, and J. H. L. Rousseau, 2005, The applicability of dip moveout/azimuth moveout in the presence of caustics: Geophysics, 70, no. 1, S1-S17, doi:10.1190/1.1852785.

Mittet, R., 2002, Multiple suppression by prestack reverse time migration: A nail in the coffin: EAGE Expanded abstracts.

2006, The behaviour of multiples in reverse-time migration schemes: EAGE Expanded abstracts.

Sava, P., and I. Vasconcelos, 2009, Efficient computation of extended images by wavefield-based migration: SEG Technical Program Expanded Abstracts, 28, 2824-2828.

Snieder, R., K. Wapenaar, and U. Wegler, 2007, Unified Green's function retrieval by cross-correlation; connection with energy principles: Physical Review E: Statistical, Nonlinear, and Soft Matter Physics, 75, no. 3, 036103, doi:10.1103/PhysRevE.75.036103. PubMed

Stolk, C. C., M. V. de Hoop, and T. J. P. M. O. Root, 2009a, Inverse scattering of seismic data in the reverse time migration (RTM) approach: GMIG report 09-06, Purdue University.

Stolk, C. C., M. V. de Hoop, and W. W. Symes, 2009b, Kinematic s of shot-geophone migration: Geophysics, 74, no. 6, WCA19-WCA34, doi:10.1190/1.3256285.

Thorbecke, J., and K. Wapenaar, 2007, On the relation between seismic interferometry and the migration resolution function: Geophysics, 72, no. 6, T61-T66, doi:10.1190/1.2785850.

van Manen, D.-J., A. Curtis, and J. O. A. Robertsson, 2006, Interferometric modeling of wave propagation in inhomogeneous elastic media using time reversal and reciprocity: Geophysics, 71, no. 4, SI47-SI60, doi:10.1190/1.2213218. 
Vasconcelos, I., 2008, Generalized representations of perturbed fields

- applications in seismic interferometry and migration: SEG Technical Program Expanded Abstracts, 27, 2927-2931.

Vasconcelos, I., P. Sava, and H. Douma, 2009, Wave-equation extended images via image-domain interferometry: SEG Technical Program Expanded Abstracts, 28, 2839-2843.

Vasconcelos, I., R. Snieder, and B. Hornby, 2008, Imaging internal multiples from subsalt vsp data examples of target-oriented interferometry: Geophysics, 73, no. 4, S157-S168, doi:10.1190/1.2944168.

Wapenaar, K., 2009, Se ismic interferometry, the optical theorem and a non-linear point diffractor : SEG Technical Program Expanded Abstracts, 28, 3595-3600.

Wapenaar, K., and J. Fokkema, 2006, Green's function representations for seismic interferometry: Geophysics, 71, no. 4, SI33-SI46, doi:10.1190/1.2213955

Weglein, A., F. B. Ara'ujo, P. M. Carvalho, R. H. Stolt, K. H. Matson, R. T. Coates, D. Corrigan, D. J. Foster, S. A. Shaw, and H. Zhang, 2003, Inverse scattering series and seismic exploration: Inverse Problems, 19, R27-R83. 